\title{
Sensory abnormalities and masticatory function after microvascular decompression or balloon compression for trigeminal neuralgia compared with carbamazepine and healthy controls
}

\author{
Michelle Cristina Ichida, MD, ${ }^{1}$ Antonio Nogueira de Almeida, MD, PhD, ${ }^{5}$ \\ Jose Claudio Marinho da Nobrega, MD, MSc, ${ }^{1,5}$ Manoel Jacobsen Teixeira, MD, PhD, ${ }^{1,2}$ \\ José Tadeu Tesseroli de Siqueira, DDS, PhD, ${ }^{3}$ and Silvia R. D. T. de Siqueira, DDS, PhD ${ }^{4}$ \\ ${ }^{1}$ Neurology Department, Medical School, University of São Paulo; ${ }^{2}$ Interdisciplinary Pain Center of Hospital das Clinicas, \\ Neurology Division, Central Institute of Hospital das Clinicas Medical School, University of São Paulo; ${ }^{3}$ Orofacial Pain Team, \\ Dentistry Division, Central Institute, Hospital das Clínicas, Medical School, University of São Paulo; ${ }^{4}$ School of Arts, Science, and \\ Humanities, University of São Paulo, and Research Group of Oral Medicine and Neuroscience of the Dentistry Division, Institute \\ of Psychiatry, Hospital das Clinicas, Medical School, University of São Paulo; and ${ }^{5}$ Functional Neurosurgery Division, Institute of \\ Psychiatry, Hospital das Clinicas, Medical School, University of São Paulo, Brazil
}

OBJECT Idiopathic trigeminal neuralgia (iTN) is a neurological condition treated with pharmacotherapy or neurosurgery. There is a lack of comparative papers regarding the outcomes of neurosurgery in patients with iTN. The objective of this study was to investigate sensory thresholds and masticatory function in 78 patients with iTN who underwent microvascular decompression (MVD) or balloon compression (BC), and compare these treatments with carbamazepine and 30 untreated healthy controls.

METHODS The authors conducted a case-controlled longitudinal study. Patients were referred to 1 of 3 groups: MVD, $\mathrm{BC}$, or carbamazepine. All patients were evaluated before and after treatment with a systematic protocol composed of a clinical orofacial questionnaire, Research Diagnostic Criteria for temporomandibular disorders, Helkimo indices, and a quantitative sensory-testing protocol (gustative, olfactory, cold, warm, touch, vibration, superficial, and deep pain thresholds).

RESULTS Both MVD and BC were effective at reducing pain intensity $(p=0.012)$ and carbamazepine doses $(p<$ $0.001)$. Myofascial and articular complaints decreased in both groups $(p<0.001)$, but only the patients in the MVD group showed improvement in Helkimo indices $(p<0.003)$. Patients who underwent MVD also showed an increase in sweet $(p=0.014)$ and salty $(p=0.003)$ thresholds. The sour threshold decreased $(p=0.003)$ and cold and warm thresholds increased $(p<0.001)$ in patients after MVD and BC, but only the patients who underwent BC had an increase in touch threshold $(p<0.001)$

CONCLUSIONS Microvascular decompression and BC resulted in a reduction in myofascial and jaw articular complaints, and the impact on masticatory function according to Helkimo indices was greater after BC than MVD. MVD resulted in more gustative alterations, and both procedures caused impairment in thermal thresholds (warm and cold). However, only BC also affected touch perception. The sensorial and motor deficits after BC need to be included as targets directly associated with the success of the surgery and need to be assessed and relieved as goals in the treatment of iTN.

http://thejns.org/doi/abs/10.3171/2014.9.JNS14346

KEY WORDS masticatory function; quantitative sensory testing; trigeminal neuralgia; orofacial pain; temporomandibular disorders; carbamazepine; balloon compression; microvascular decompression; functional neurosurgery

ABBREVIATIONS BC = balloon compression; IASP = International Association for the Study of Pain; iTN = idiopathic TN; MVD = microvascular decompression; RDC = Research Diagnostic Criteria; TMD = temporomandibular disorder; TN = trigeminal neuralgia; $\mathrm{VAS}=$ visual analog scale.

SUBMITTED February 21, 2014. ACCEPTED September 11, 2014.

INCLUDE WHEN CITING Published online April 3, 2015; DOI: 10.3171/2014.9.JNS14346.

DISCLOSURE This study was developed with the financial support of FAPESP (Research Foundation of São Paulo, grant no. 2010/01620-4). 
I DIOPATHIC trigeminal neuralgia (iTN) is a neuropathic pain that affects 1 or more branches of the trigeminal nerve. The diagnostic criteria of iTN are shock-like pain (lasting from a few milliseconds to approximately 2 minutes), trigger points or spontaneous pain, strong intensity, and absence of pain between crises. ${ }^{15,17}$ Despite the good performance of pharmacological treatment for iTN, ${ }^{2}$ approximately $75 \%$ of patients need neurosurgical intervention. ${ }^{4}$ This intervention can alter sensory and masticatory functions, but these abnormalities have not been detailed or compared according to the surgical procedure used. ${ }^{10,15}$

Microvascular decompression (MVD) is an open surgery that was introduced by Peter Jannetta in $1967 .{ }^{33}$ It is considered to have the best long-term results for iTN, with a low rate of recurrence of pain $(15 \%-25 \%$ within 3 years $)^{13,23}$ and few collateral effects., ${ }^{1,4,20-22,26}$ The percutaneous balloon compression (BC) of the trigeminal ganglion was introduced by Mullan and Lichtor in 1983. 3,4,32 The recurrence rate after $\mathrm{BC}$ is $45.4 \%$ in 4 years, ${ }^{19}$ and there is a high incidence of masticatory complications ${ }^{5}$ and sensory deficits ${ }^{9,10,29,30}$ after this procedure. Other treatment options for iTN include stereotactic radiosurgery using the Gamma Knife, radiofrequency thermal rhizotomy, and botulinum toxin injections at the trigger zone. ${ }^{4,13,20,21}$

According to the scientific literature, MVD appears to cause fewer sensory alterations than other treatments, although it is more often related to rare but serious complications. However, to our knowledge, there are no studies comparing these techniques in a prospective design, including complete examination of the orofacial area with detailed sensory and functional quantification. Thus, the objective of this study was to investigate the sensory abnormalities and functional masticatory findings in patients with iTN who underwent surgery (MVD or BC) compared with carbamazepine-treated patients and healthy controls. The functional evaluation included instruments to investigate the masticatory function, orofacial and pain characteristics, local and systemic comorbidities and associated factors, and a systematic protocol for quantitative sensory testing at the orofacial region.

\section{Methods \\ Study Population}

In this prospective study, we enrolled 78 patients who received a diagnosis of $\mathrm{iTN}$ according to the International Association for the Study of Pain (IASP) criteria ${ }^{17}$ and were referred from the Orofacial Pain Clinic of the Dentistry Division and the Facial Pain Ambulatory Clinic of the Neurology Division, Hospital das Clinicas, School of Medicine, University of São Paulo, Brazil. All patients had been treated with anticonvulsants and had been receiving between 600 and $1200 \mathrm{mg} /$ day of carbamazepine in the prior 6 months. The patients were evaluated at the Neurosurgery Division of the Psychiatric Institute of the same hospital. Thirty age- and sex-matched healthy controls were also included. These subjects were employees of the hospital or relatives and should not have had any orofacial or body pain complaint.

All patients and controls were informed about the purposes of the study and all signed the informed consent form. The protocol had been approved by the local ethics committee. The exclusion criteria for iTN patients were symptomatic neuralgia (based on clinical evaluation and imaging exams, i.e., CT and/or nuclear MRI), multiple sclerosis or other neurological diseases, and rheumatologic systemic diseases.

\section{Group Distribution}

The patients were classified using IASP criteria into 1 of 3 groups according to the treatment that was indicated: 1) MVD (18 patients), 2) BC (30 patients), and 3) carbamazepine (30 patients). The patients referred to MVD were selected according to their ages and lack of comorbidities, after the evaluation performed by an experienced neurosurgeon. These patients were treated according to principles outlined by Jannetta. ${ }^{16}$ After general anesthesia, the patients were positioned with their heads turned to the side opposite to the pain and a straight incision behind the ear was made. A skull portion of approximately $25-30 \mathrm{~mm}$ in diameter was removed, and the dura was opened. The dissection was performed with a microscope to expose the trigeminal nerve. The blood vessel was mobilized and the nerve decompressed. The procedure was performed in all patients by only 1 neurosurgeon (J.C.M.N.). All patients completed the 6-month evaluation period.

The patients in the $\mathrm{BC}$ group underwent the technique according to Brown and Mullan. ${ }^{5}$ After sedation with midazolam and propofol, and the anesthetic trigeminal nerve block with 2\% lidocaine, a Fogarty catheter was percutaneously introduced with a balloon through the foramen ovale to reach the trigeminal ganglion, where it was inflated to form a pear shape, kept in place for a minute, and then removed. Radiology confirmed the position of the balloon. The procedure was performed in all patients by only 1 neurosurgeon (J.C.M.N.). All patients completed the 6-month evaluation period.

Thirty patients who had been treated with 600-1200 $\mathrm{mg}$ of carbamazepine per day for at least 6 months were included in the third group. Thirty healthy age- and sexmatched subjects were included in the control group.

Demographic data were compared using Pearson's chisquare test (SPSS version 17.0, SPSS Inc.). There were statistically significant differences in ages $(p=0.006$, chi-square test) but not in sex ( $p=0.965,1$-way ANOVA). The patients in the MVD group were younger ([mean \pm SD] $49.17 \pm 13.16$ years old) than in the other groups. The mean age of the patients in the BC group was 61.97 \pm 10.98 years, compared with $60.83 \pm 11.08$ years in the carbamazepine group and $60.57 \pm 13.54$ years in the control group.

The patients and the evaluator could not be blinded to the treatments due to the easy possibility of identification (e.g., area of surgical approach). A control group of healthy subjects free of medication and pain was included because discrete sensory deficits can occur in the natural history of iTN even in patients who do not undergo surgery. In addition, carbamazepine itself acts on the nervous system and can cause sensory abnormalities.

\section{Evaluation Schedule}

The patients forming the MVD and BC groups were 
evaluated according to the following protocol: 1) before treatment (within 60 days); 2) immediately after the treatment (between 6 and 8 days, mean 7 days); 3) 30 days after the treatment ( \pm 7 days); and 4) 6 months after the treatment ( \pm 14 days). The patients in the carbamazepine and control groups were evaluated at 2 different time points, at initial evaluation and then a reevaluation after 6 months. The patients in the carbamazepine and control groups were reevaluated to avoid fluctuations in sensitivity and masticatory abnormalities during the period of study. The means between both evaluations of these groups were considered in the analysis.

\section{Instruments of Evaluation}

The entire sample was evaluated by the same examiner (M.C.I.), previously trained to use testing tools calibrated by the Orofacial Pain Group of the hospital. Several evaluation tools were used.

The first tool was a clinical evaluation protocol from the Division of Dentistry, Orofacial Pain Clinic, and Group of Oral Medicine and Neuroscience. This protocol consisted of a main complaint, pain characteristics (location, duration, descriptors, intensity according to the visual analog scale [VAS], and causal, alleviation, and aggravation factors); medical history and medications; presence of earache, headache, generalized body pain; and the complete orofacial and dental examination. ${ }^{8}$ This instrument allowed the assessment of all orofacial characteristics or complaints present in these patients.

The second tool was Research Diagnostic Criteria (RDC) for temporomandibular disorders (TMDs), Axes I and II (validated version in Portuguese) ${ }^{7,12}$ This tool consists of a protocol for the diagnosis of TMDs, which are myofascial, articular disc, and articular bone diseases of the masticatory system (Axis I), and the evaluation of associated emotional and functional aspects including depression, anxiety symptoms, and mandibular limitations (Axis II).

The third tool was an evaluation of joint, muscular, and orofacial occlusion by Helkimo indices validated for the Portuguese language. ${ }^{14}$ This tool consists of a questionnaire that rates the dental occlusion, mandibular movements and their limitations, and clinical dysfunction of and complaints about the masticatory function.

The final tool used was quantitative sensory testing. All participants in the study underwent a standardized protocol of quantitative sensory testing, which consists of tests grouped as follows: ${ }^{11}$ gustative and olfactory thresholds, thermal detection thresholds for cold and warm sensations, mechanical detection thresholds for touch and vibration perception, and mechanical pain sensitivity, including superficial and deep pain thresholds.

The facial areas evaluated were the 3 trigeminal branches (front, cheek, and chin). The evaluation was performed bilaterally in all participants. All patients were evaluated in the sitting position, with the head resting on a flat surface, and in a silent room with acoustic sealing on the walls and the door closed. Only the patient and the researcher were in the room. The same researcher evaluated all participants. The patients and controls were instructed to keep their eyes closed during the examination and to concentrate on the stimuli applied. Only the researcher knew the order of the stimuli.

\section{Gustative Thresholds}

For gustative thresholds,,$^{11}$ the following 4 substances, corresponding to the 4 tastes, were tested. For each test, 1 drop was applied to the tongue, starting with the lowest concentration, interleaved with 1 drop of distillate water, and the concentration was increased until the participant detected and identified the stimulus. The 4 substances and concentrations used were: 1) sweet (glucose) at $0.01 \mathrm{M}$, $0.032 \mathrm{M}, 0.1 \mathrm{M}, 0.32 \mathrm{M}$, and $1.0 \mathrm{M} ; 2$ ) salty (sodium chlorate) at $0.01 \mathrm{M}, 0.032 \mathrm{M}, 0.1 \mathrm{M}, 0.32 \mathrm{M}$, and $1.0 \mathrm{M} ; 3$ ) sour (citric acid) at $0.00032 \mathrm{M}, 0.001 \mathrm{M}, 0.0032 \mathrm{M}, 0.01 \mathrm{M}$, and $0.032 \mathrm{M}$; and 4) bitter (urea) at $0.1 \mathrm{M}, 0.32 \mathrm{M}, 1.0 \mathrm{M}, 3.2$ $\mathrm{M}$, and $10.0 \mathrm{M}$.

\section{Olfactory Thresholds}

For olfactory thresholds, ${ }^{11}$ the participants were evaluated with isopropanol solutions in polyethylene bottles interleaved with distillate water, starting with the lowest concentration and increasing until the participant detected the stimulus: $0.09 \%, 13.0 \%, 23.0 \%, 35.0 \%, 53.0 \%$, and $70.0 \%$.

\section{Thermal Detection}

Thermal testing was performed using the MSA thermo test device (Somedic). The baseline temperature was $32^{\circ} \mathrm{C}$ and the contact square area of the thermode was $9 \times 9$ $\mathrm{mm}$. Cold detection threshold and warm detection threshold were assessed using ramped stimuli at $1^{\circ} \mathrm{C} / \mathrm{sec}$. The evaluation consisted of 5 measurements for each thermal threshold, and the means and standard deviations were considered for the analysis.

\section{Mechanical Detection Threshold}

Touch perception was assessed using a set of standardized von Frey filaments with rounded tips $0.5 \mathrm{~mm}$ in diameter, applied with an electronic device (IITC). Three measurements in $\mathrm{g} / \mathrm{mm}^{2}$ were performed and the means and standard deviations were considered for the analysis.

\section{Vibration Detection Threshold}

Vibration testing was performed using the electronic Vibrameter device (Somedic) with a vibrator weighing $650 \mathrm{~g}$ and a contact area of $1 \mathrm{~cm}^{2}$, perpendicularly applied to the skin for the threshold detection using ramped stimuli at $1 \mathrm{~Hz} / \mathrm{sec}$. The method of calculating the vibration threshold consisted of the mean between the appearance and disappearance thresholds detected by the patient.

\section{Pressure Pain Perception}

Deep algometry was measured with the electronic pressure algometer (Somedic) with a probe area of $1 \mathrm{~cm}^{2}$ that was pressed on the skin with a ramp rate of $50 \mathrm{kPa} / \mathrm{sec}$.

\section{Superficial Pain Perception}

Superficial algometry was tested using disposable needles of $8 \times 10 \times 0.5 \mathrm{~mm}$, applied with an electronic device 
(IITC). Three measurements in $\mathrm{g} / \mathrm{mm}^{2}$ were performed and the means and standard deviations were considered for the analysis.

\section{Statistical Analysis}

All data were tabulated and the frequencies and percentages, means, standard deviations, and ranges were compared between the groups. After the initial descriptive evaluation, variables were tested for normal distribution with the Shapiro-Wilk test and Q-Q plots. For quantitative variables with normal distribution we used 1-way ANOVA and analysis of repetitive measures. Post hoc comparisons were calculated using the Tukey test. Nonparametric tests included the Pearson's chi-square, Fisher's exact, and McNemar tests. All statistical calculations were performed using SPSS (version 17.0, SPSS Inc.). The level of significance was $5 \%$.

\section{Results \\ Initial Comparison and Group Matching}

Hypertension was observed in $50.1 \%$ of the sample, and diabetes in $10.2 \%$ ( $p=0.129$, chi-square test). The groups did not significantly differ according to medication in use ( $p>0.05$, chi-square test).

The right side (40.7\%) and the mandibular branch were the most affected (30.8\%), with no statistical differences between the groups (side: $p=0.191$, chi-square test; branch: $p=0.528$ ). The mean duration of the disease was $6.0 \pm 5.4$ years $(\mathrm{p}=0.326,1$-way ANOVA). Forty-five percent of the patients reported associated causal factors (dental treatments, stress, and others). Pain was primarily relieved with medication (61.5\%); cold weather (35.9\%) and emotional stress $(28.2 \%)$ were aggravating factors ( $\mathrm{p}$ $>0.05$, chi-square test). According to the VAS, the mean pain intensity was $6.0 \pm 5.4(\mathrm{p}=0.519$, 1 -way ANOVA). Some patients had low pain intensity because all of them were being treated with medication, which could have partially or completely alleviated the symptoms. The most common pain descriptor was shock-like pain $(88.5 \%$; $\mathrm{p}=$ 0.05 , chi-square test). Table 1 shows the pain characteristics of each group at the initial evaluation. The patients referred to neurosurgery had twice as much pain intensity according to the VAS than the patients who continued pharmacological treatment.

There were no significant differences between the groups in relation to dental characteristics ( $p=0.137$, chisquare test). Seventy percent of this sample was edentulous. The $\mathrm{BC}$ group had a greater loss of width in the vertical dimension due to edentulism $(40.0 \%)$ than the other groups, and $25 \%$ of the sample had abnormal occlusion (open bite, cross bite, or deep bite). Periodontal disease was observed in 8 participants (7.4\%). The study groups (MVD, BC, and carbamazepine) did not differ in the diagnosis of myofascial pain $(\mathrm{p}=0.171$, chi-square test $)$, disc displacements ( $\mathrm{p}=0.731$, chi-square test), and articular diseases of the temporomandibular joint ( $\mathrm{p}=0.545$, chisquare test) according to the RDC for TMDs. The control group had more bilateral chewing $(\mathrm{p}<0.002$, chi-square test), less myofascial pain ( $\mathrm{p}=0.004$, chi-square test), less masticatory discomfort ( $\mathrm{p}<0.001$, chi-square test), and higher maximum mouth opening $(\mathrm{p}=0.012)$ than the other groups.

\section{Longitudinal Comparison}

Table 1 shows the comparison of pain characteristics between the groups at different periods of evaluation. The mean pain intensity of the patients referred to neurosurgery (both MVD and BC) was significantly higher than the mean pain intensity of the patients pharmacologically treated. There was a significant reduction in the pain intensity in both groups (MVD and BC) after the surgical procedure $(\mathrm{p}=0.012)$, which was characterized by pain alleviation. However, none of the patients in the BC group had recurrence of pain compared with 2 patients (11.1\%) in the MVD group with pain recurrence (no statistical difference). The number of patients with pain descriptors also decreased after both surgical procedures $(p<0.001)$ as well as carbamazepine doses $(\mathrm{p}<0.001)$.

Myofascial pain complaints and articular abnormalities associated with TMDs were observed in $18.2 \%-43.3 \%$ of the patients in this sample. A reduction in these complaints was noted at the postoperative evaluation conducted 6 months after the surgical procedure in both the MVD and $\mathrm{BC}$ groups $(\mathrm{p}<0.001)$. A decrease in all the Helkimo indices occurred only after MVD ( $<<0.003$; Table 2).

There were no significant differences between the MVD and BC groups related to improvement in emotional and quality of life indices of RDC. Both groups had a significant reduction in the degree of pain severity $(\mathrm{p}<$ $0.001)$, depression trait $(p=0.006)$, and mean of mandibular limitations $(\mathrm{p}<0.001$; Table 3$)$.

The following significant differences in the gustative thresholds were observed: high sweet thresholds at the postoperative evaluation after MVD compared with the baseline value and controls ( $\mathrm{p}=0.014)$; high salty thresholds at the 7- and 30-day postoperative evaluations after MVD compared with the baseline value and controls $(\mathrm{p}=$ 0.003); and decrease in the sour threshold at the 6-month postoperative evaluation compared with the baseline value and controls $(p=0.003$; Table 4$)$. The somatosensory evaluation showed significantly higher cold and warm thresholds at the postoperative evaluations (MVD and BC groups) compared with the controls $(\mathrm{p}<0.001)$; significantly higher touch thresholds at the 30-day postoperative evaluation after BC compared with the other groups ( $p<$ 0.001); significantly lower vibration thresholds after MVD and $\mathrm{BC}$ compared with the carbamazepine and control groups $(\mathrm{p}<0.001)$; and significantly higher cold, warm, and touch thresholds in all patient groups compared with controls ( $\mathrm{p}<0.001$; Table 5).

\section{Discussion}

In this study, both MVD and BC were effective for pain relief, reducing the mean pain intensity, pain descriptors, and carbamazepine doses. ${ }^{3,25}$ Both groups showed similar results for emotional and quality of life variables, reducing the degree of severity, depression index, and limitations in mandibular function $(\mathrm{p}<0.001)$ after the surgery. However, the masticatory function and the mechanical (touch) perception were better preserved after MVD than 


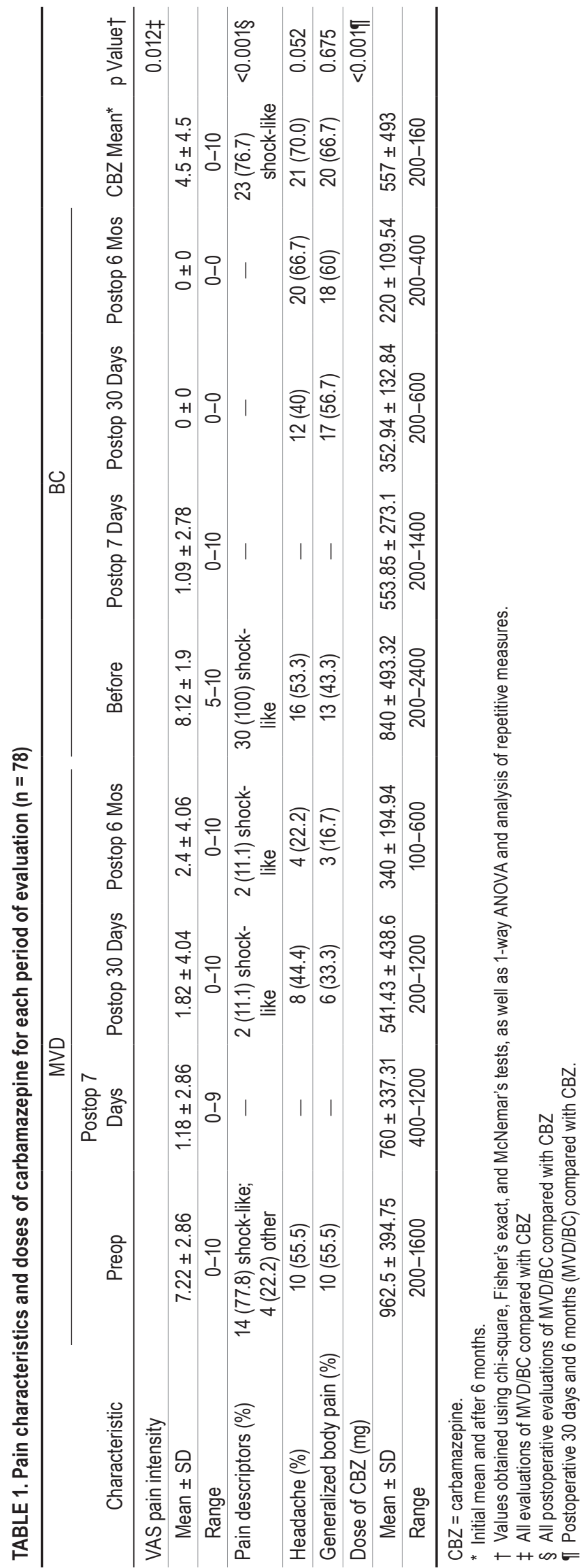

BC. There is a lack of comparative studies that have analyzed the outcomes of different types of neurosurgery for iTN, and despite the fact that there are clear criteria for the selection of surgical modalities, ${ }^{18,24,26}$ for a great number of patients this choice is made according to the available technique.

Historically, sensorial and motor deficits with a neurological etiology that occurred as complications of these neurosurgical modalities have been investigated, as well as their associations with pain alleviation and the success of these procedures. However, there is also an impact of the surgeries on the functionality of the masticatory system not directly associated with the neurological deficit. ${ }^{10}$ There is an operative impact on the pterygoideal area (and pterygoid muscles) that affects the mouth's mobility. In addition, sensory deficits can alter taste and smell perception by CNS processing and can severely affect the sensory input necessary for the perception for food, saliva, and dental prosthesis in the mouth, causing impairment in mastication, swallowing, talking, social participation, and quality of life. ${ }^{9,29,30}$ Avoiding these should be a goal in iTN treatment; it is certainly part of the goals in the treatment for any chronic pain condition.

The orofacial tissues are directly and indirectly involved in any type of facial pain, including iTN. In this case-controlled longitudinal study, myofascial and articular symptoms in the masticatory system were reduced after MVD and BC and maintained at a lower level after 6 months. However, the masticatory function according to Helkimo indices improved only after MVD. This technique has less impact on orofacial tissues, whereas BC perforates the pterygoid muscles and can cause limited mouth opening..$^{10}$ Thus, the impact on the facial region is minor in MVD, and the presence of mandibular abnormalities should be considered a risk factor in the outcomes of BC.

Myofascial pain is a common consequence of pain chronification (development of pain memory and persistence of pain for more than 3-6 months), and it can become a comorbidity needing adequate treatment. ${ }^{10}$ Its mechanisms involve neurons from the trigeminal complex nuclear system and other adjacent areas that act as premotors of masticatory muscular groups, offering neural substratum for the sensory control of the masticatory function. ${ }^{11}$ That is the reason why the control group had less myofascial pain, less masticatory discomfort, and wide maximum mouth opening. The presence of myofascial pain in these patients supports the idea that the central sensitization process is induced by iTN in the trigeminal complex; it aims at maintaining a protective mandibular position to avoid injury. ${ }^{11}$

There was an increase in sweet and salty thresholds after MVD and a decrease in sour thresholds after both types of surgery. MVD generated more abnormalities in gustative thresholds in this study. Since the beginning of the 20th century, taste alteration has been observed after TN surgery. It has been more recently supported by many clinical articles showing abnormal gustative and olfactory functions in patients with orofacial neuropathic pain. ${ }^{11,31}$ There is ample evidence of sensorial interaction between the trigeminal gustative and olfactory systems, which can 
TABLE 2. Temporomandibular disorders according to RDC and Helkimo indices for each period of evaluation $(n=108)$

\begin{tabular}{|c|c|c|c|c|c|c|c|c|c|}
\hline \multirow[b]{2}{*}{ Diagnosis (questionnaire) } & \multicolumn{3}{|c|}{ MVD (\%) } & \multicolumn{3}{|c|}{$\mathrm{BC}(\%)$} & \multirow[b]{2}{*}{$\begin{array}{l}\text { CBZ Mean } \\
(\%)^{*}\end{array}$} & \multirow[b]{2}{*}{$\begin{array}{c}\text { Control Mean } \\
(\%)^{*}\end{array}$} & \multirow[b]{2}{*}{ p Value } \\
\hline & Preop & $\begin{array}{c}\text { Postop } 30 \\
\text { Days }\end{array}$ & $\begin{array}{c}\text { Postop } 6 \\
\text { Mos }\end{array}$ & Preop & $\begin{array}{c}\text { Postop } 30 \\
\text { Days }\end{array}$ & $\begin{array}{c}\text { Postop } 6 \\
\text { Mos }\end{array}$ & & & \\
\hline Myofascial pain (RDC/TMD) & $7(38.9)$ & $3(16.7)$ & $4(22.2)$ & $6(20)$ & $7(23.3)$ & $3(10)$ & $8(26.7)$ & $0(0.0)$ & $<0.001 \ddagger$ \\
\hline Disc displacement (RDC/TMD) & $3(16.7)$ & $2(11.1)$ & $1(5.5)$ & $3(10)$ & $3(10)$ & $3(10)$ & $2(6.7)$ & $1(3.3)$ & $<0.001 \S$ \\
\hline Articular disease (RDC/TMD) & $6(33.3)$ & $2(11.1)$ & $2(11.1)$ & $13(43.3)$ & $13(43.3)$ & $7(23.3)$ & $17(56.7)$ & $9(30.0)$ & $<0.001 \ddagger$ \\
\hline Anamnestic dysfunction (Helkimo) & $15(83.3)$ & $3(16.7)$ & $6(33.3)$ & $19(63.3)$ & $15(50)$ & $14(46.7)$ & $19(63.3)$ & $7(23.3)$ & $<0.001 \rrbracket$ \\
\hline Clinical dysfunction (Helkimo) & $14(77.7)$ & $5(27.8)$ & $6(33.3)$ & $23(76.7)$ & $24(80)$ & $15(50)$ & $23(76.7)$ & $14(46.7)$ & $<0.001 \ddagger$ \\
\hline Mandibular mobility (Helkimo) & $16(88.8)$ & $9(50)$ & $8(44.4)$ & $13(43.3)$ & $11(36.7)$ & $8(26.7)$ & $12(40.0)$ & $17(56.7)$ & $<0.001^{* *}$ \\
\hline Occlusion dysfunction (Helkimo) & $16(88.8)$ & $7(38.9)$ & $6(33.3)$ & $14(46.7)$ & $14(46.7)$ & $12(40)$ & $11(36.7)$ & $14(46.7)$ & $0.003 \S$ \\
\hline
\end{tabular}

* Initial mean and after 6 months.

$\dagger$ Values obtained using the chi-square, Fisher's exact, and McNemar tests.

$\ddagger$ Before MVD/BC and CBZ compared with controls.

$\S$ Before MVD compared with CBZ and controls.

If Before MVD compared with 6-month postoperative MVD and controls.

** Before MVD/BC compared with 6-month postoperative MVD/BC

be demonstrated by the convergence of inputs at the solitary tract, at the trigeminal complex, and in other subcortical areas.

MVD is known as a procedure with little impact on facial sensitivity and few numbness complaints. This study supports these characteristics of MVD by the increase in touch thresholds only in the group of patients who underwent BC. However, both neurosurgical procedures resulted in an increase in cold and warm thresholds. This result can be attributed to neuroplastic phenomena involving subcortical areas related to sensorial interaction, the same mechanisms that underlie the alteration in taste after neurosurgery. ${ }^{30}$ The reason why MVD did not produce abnormal mechanical thresholds is not clear and needs further investigation. One possible explanation is the distribution of mechanical nerve fibers in the trigeminal root, which possibly makes small fibers (such as pain and thermal) more prone to injuries than the large ones. This could be, at least in part, the mechanism underlying the effect of MVD on iTN pain.

In the past, it was a universally understood concept that there were no abnormalities in the neurological examination of patients with iTN. More recent studies have shown that discrete tactile and thermal hypoesthesia are present in these patients. ${ }^{6,27,33}$ In this study, this thermal hypoesthesia has also been evidenced. The pathophysiological mechanisms of iTN involve membrane abnormal processing with ectopic action potentials and sodium channel alterations located at the entry zone of the trigeminal nerve. ${ }^{28}$ This area may present compression (vascular or not) and abnormal deposits of myelin that interfere with the membrane of small fibers such as the ones related to pain and temperature, causing iTN.

MVD could be considered the surgery for iTN with better long-term results for pain and less discomfort generated by sensory impairment, which is shown by the thermal and gustative thresholds. However, when patients are being referred, this needs to be balanced against the bigger short-term risks involved, and the patients' general health status and age must also be considered. Although BC is safer for the elderly and patients with chronic diseases, its impact on orofacial sensation and function should be measured, assessed, and noted as one of the priorities in the treatment for iTN. Particularly in the period after BC, the recurrence of pain needs to be assessed according to the history of the patients, masticatory limitations, and pain descriptors to determine whether their pain remains iTN related or a musculoskeletal problem due to BC. Patients

TABLE 3. Emotional evaluation by the RDC Axis II for each period of evaluation $(n=108)$

\begin{tabular}{|c|c|c|c|c|c|c|c|c|c|}
\hline \multirow[b]{2}{*}{ Emotional Evaluation } & \multicolumn{3}{|c|}{ MVD } & \multicolumn{3}{|c|}{$\mathrm{BC}$} & \multirow[b]{2}{*}{ CBZ Mean* } & \multirow[b]{2}{*}{$\begin{array}{l}\text { Control } \\
\text { Mean* }\end{array}$} & \multirow[b]{2}{*}{$p$ Value } \\
\hline & Preop & $\begin{array}{l}\text { Postop } 30 \\
\text { Days }\end{array}$ & $\begin{array}{l}\text { Postop } 6 \\
\text { Mos }\end{array}$ & Preop & $\begin{array}{l}\text { Postop } 30 \\
\text { Days }\end{array}$ & $\begin{array}{l}\text { Postop } 6 \\
\text { Mos }\end{array}$ & & & \\
\hline $\begin{array}{l}\text { Mean degree of pain severity } \\
\pm S D\end{array}$ & $3.06 \pm 1.0$ & $0.73 \pm 1.6$ & $1.2 \pm 1.93$ & $2.91 \pm 0.5$ & $0.7 \pm 0.36$ & $0 \pm 0$ & $1.9 \pm 1.12$ & $0 \pm 0$ & $<0.001 \ddagger$ \\
\hline Depression (\%) & $13(72.2)$ & $7(38.9)$ & $3(16.7)$ & $25(83.3)$ & $15(50)$ & $17(56.7)$ & $14(46.7)$ & $9(30.0)$ & $0.006 \ddagger$ \\
\hline Physical symptoms (\%) & $13(72.2)$ & $7(38.9)$ & $4(22.2)$ & $25(83.3)$ & $15(50)$ & $19(63.3)$ & $13(43.3)$ & $14(46.7)$ & $0.392 \ddagger$ \\
\hline $\begin{array}{l}\text { Mean mandibular limitations } \\
\quad \pm \mathrm{SD}\end{array}$ & $80.8 \pm 27$ & $15.9 \pm 35$ & $28.3 \pm 45.8$ & $82.1 \pm 29$ & $1.67 \pm 9.1$ & $0 \pm 0$ & $61.37 \pm 37.8$ & $0 \pm 0$ & $<0.001 \ddagger$ \\
\hline
\end{tabular}



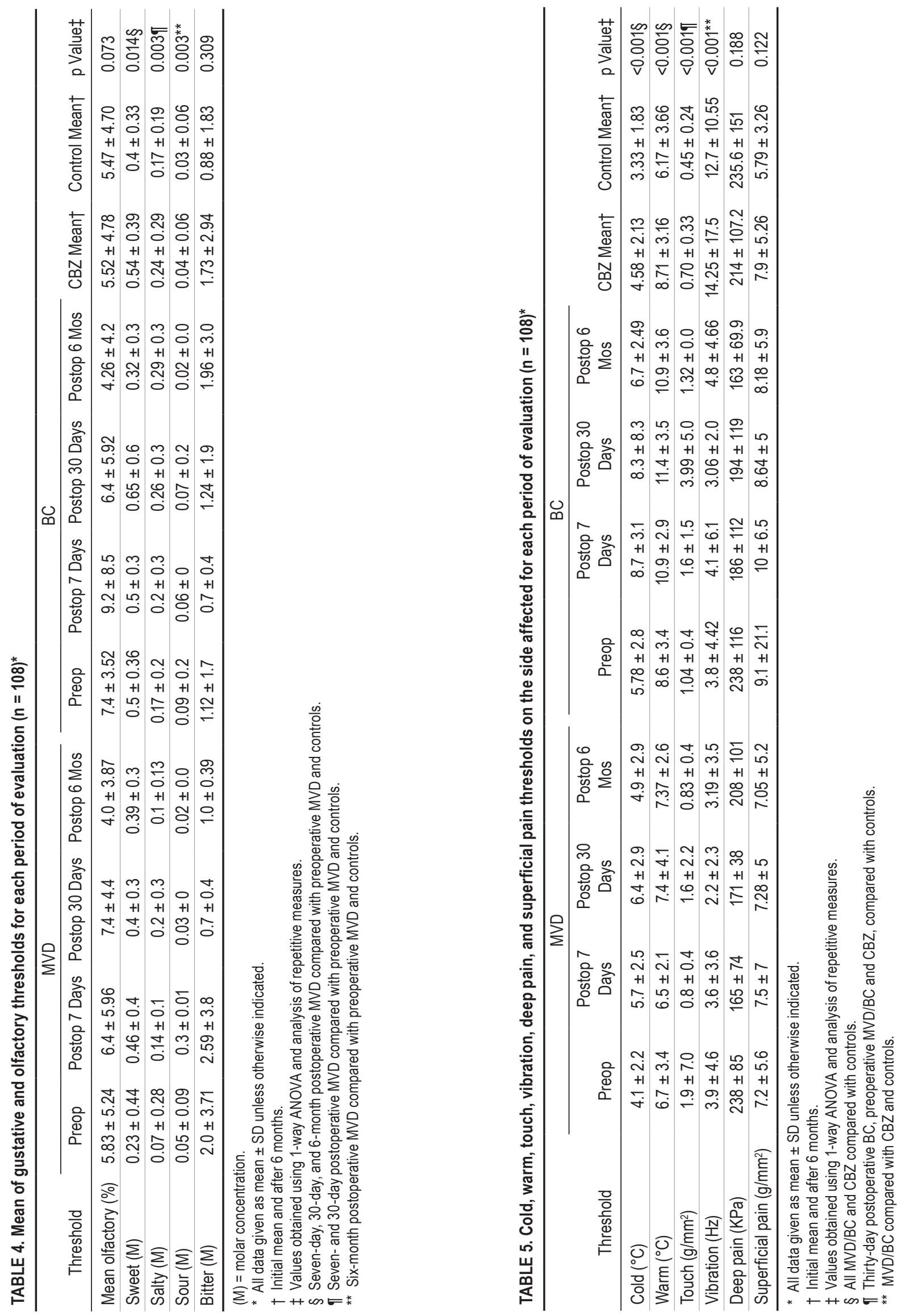
with iTN treated with medication are not free of risks, and they require periodic hematological examinations to check hepatic function and blood count. ${ }^{4}$

One limitation of this study is the impossibility of age matching. Although MVD is a safe procedure for patients of any age, ${ }^{26}$ due to more severe comorbidities in older populations the younger patients were more often referred to MVD. There are age characteristics associated with the masticatory system and sensorial thresholds that could have affected, at least in part, the results obtained by this study. The other variables that could have interfered with the analysis (prevalence of comorbidities, dental pain, and orofacial characteristics) did not present statistically significant differences between the groups. In addition, it was also not possible to make the evaluator or the patients blind to the groups, which also needs to be considered in the analysis of the results.

Another limitation is the effect of carbamazepine on the nervous system, which could alter sensorial perception. This effect was the reason for the inclusion of a control group of patients with iTN with no history of neurosurgery and pharmacotherapy-only treatment.

\section{Conclusions}

MVD and BC resulted in a decrease in myofascial and jaw articular complaints, and the impact on the masticatory function and mobility was greater after BC than MVD. Myofascial pain relief with a surgical procedure should indicate that this pain could be an effect of central sensitization by chronic pain and iTN. On the other hand, MVD resulted in more gustative alterations, and both procedures caused an increase in thermal thresholds (warm and cold), although only BC also affected touch perception. The numbness complaint of patients who underwent $\mathrm{BC}$ is more likely associated with abnormal touch perception, but MVD can also affect small fibers related to temperature and therefore also causes sensory disturbance. Despite the fact that none of the patients in the $\mathrm{BC}$ group experienced pain again after 6 months, 2 patients had pain recurrence after MVD, but this was not a statistically significant difference. The sensorial and motor deficits after $\mathrm{BC}$ need to be included as targets directly associated with the success of the surgery and need to be assessed and relieved as a goal in the treatment of iTN.

\section{References}

1. Ashkan K, Marsh H: Microvascular decompression for trigeminal neuralgia in the elderly: a review of the safety and efficacy. Neurosurgery 55:840-850, 2004

2. Attal N, Cruccu G, Baron R, Haanpää M, Hansson P, Jensen TS, et al: EFNS guidelines on the pharmacological treatment of neuropathic pain: 2010 revision. Eur J Neurol 17:1113e88, 2010

3. Baabor MG, Perez-Limonte L: Percutaneous balloon compression of the gasserian ganglion for the treatment of trigeminal neuralgia: personal experience of 206 patients. Acta Neurochir Suppl 108:251-254, 2011

4. Broggi G, Ferroli P, Franzini A: Treatment strategy for trigeminal neuralgia: a thirty years experience. Neurol Sci 29 (Suppl 1):S79-S82, 2008

5. Brown JA, Mullan S: Percutaneous trigeminal nerve compression, in Schmidek HH, Sweet WH (eds): Operative Neu- rosurgical Techniques, ed 4. Philadelphia: Saunders, 2000, pp 1552-1559

6. Casey KF: Role of patient history and physical examination in the diagnosis of trigeminal neuralgia. Neurosurg Focus 18(5):E1, 2005

7. Cavalcanti RF, Studart LM, Kosminsky M, de Goes PS: Validation of the multimedia version of the RDC/TMD axis II questionnaire in Portuguese. J Appl Oral Sci 18:231-236, 2010

8. de Siqueira JTT, Lin HC, Nasri C, de Siqueira SRDT, Teixeira MJ, Heir G, et al: Clinical study of patients with persistent orofacial pain. Arq Neuropsiquiatr 62:988-996, 2004

9. de Siqueira SRDT, da Nóbrega JCM, de Siqueira JTT, Teixeira MJ: Frequency of postoperative complications after balloon compression for idiopathic trigeminal neuralgia: prospective study. Oral Surg Oral Med Oral Pathol Oral Radiol Endod 102:e39-e45, 2006

10. de Siqueira SRDT, da Nóbrega JCM, Teixeira MJ, de Siqueira JTT: Masticatory problems after balloon compression for trigeminal neuralgia: a longitudinal study. J Oral Rehabil 34:88-96, 2007

11. de Siqueira SRDT, Teixeira MJ, de Siqueira JTT: Orofacial pain and sensory characteristics of chronic patients compared with controls. Oral Surg Oral Med Oral Pathol Oral Radiol 115:e37-e45, 2013

12. Dworkin SF, LeResche L: Research diagnostic criteria for temporomandibular disorders: review, criteria, examinations and specifications, critique. J Craniomandib Disord 6:301355,1992

13. Haridas A, Mathewson C, Eljamel S: Long-term results of 405 refractory trigeminal neuralgia surgeries in 256 patients. Zentralbl Neurochir 69:170-174, 2008

14. Helkimo M: Studies on function and dysfunction of the masticatory system. II. Index for anamnestic and clinical dysfunction and occlusal state. Sven Tandlak Tidskr 67:101-121, 1974

15. International Association for the Study of Pain: Proceedings of the 8th World Congress on Pain. Seattle: IASP Press, 1997

16. Jannetta PJ: Vascular decompression in trigeminal neuralgia, in Samii M, Jannetta PJ (eds): The Cranial Nerves. Berlin: Springer, 1981, pp 331-340

17. Merskey H, Bogduk N (eds): Classification of Chronic Pain: Descriptions of Chronic Pain Syndromes and Definitions of Pain Terms. Seattle: IASP Press, 1994

18. Montano N, Papacci F, Cioni B, Di Bonaventura R, Meglio $\mathrm{M}$ : The role of percutaneous balloon compression in the treatment of trigeminal neuralgia recurring after other surgical procedures. Acta Neurol Belg 114:59-64, 2014

19. Omeis I, Smith D, Kim S, Murali R: Percutaneous balloon compression for the treatment of recurrent trigeminal neuralgia: long-term outcome in 29 patients. Stereotact Funct Neurosurg 86:259-265, 2008

20. Pagni CA, Fariselli L, Zeme S: Trigeminal neuralgia. Noninvasive techniques versus microvascular decompression. It is really available any further improvement? Acta Neurochir Suppl 101:27-33, 2008

21. Pollack IF, Jannetta PJ, Bissonette DJ: Bilateral trigeminal neuralgia: a 14-year experience with microvascular decompression. J Neurosurg 68:559-565, 1988

22. Pollock BE, Foote RL, Stafford SL, Link MJ, Gorman DA, Schomberg PJ: Results of repeated gamma knife radiosurgery for medically unresponsive trigeminal neuralgia. J Neurosurg 93 (Suppl 3):162-164, 2000

23. Sandell T, Eide PK: Effect of microvascular decompression in trigeminal neuralgia patients with or without constant pain. Neurosurgery 63:93-100, 2008

24. Sarnvivad P, Bumpenboon A, Chumnanvej S: Retrospective long term outcome following microvascular decompression 
surgery in Thai patients with trigeminal neuralgia. J Med Assoc Thai 96:801-806, 2013

25. Sarsam Z, Garcia-Fiñana M, Nurmikko TJ, Varma TR, Eldridge P: The long-term outcome of microvascular decompression for trigeminal neuralgia. Br J Neurosurg 24:18-25, 2010

26. Sekula RF Jr, Frederickson AM, Jannetta PJ, Quigley MR, Aziz KM, Arnone GD: Microvascular decompression for elderly patients with trigeminal neuralgia: a prospective study and systematic review with meta-analysis. J Neurosurg 114:172-179, 2011

27. Sinay VJ, Bonamico LH, Dubrovsky A: Subclinical sensory abnormalities in trigeminal neuralgia. Cephalalgia 23:541544,2003

28. Siqueira SRDT, Alves B, Malpartida HMG, Teixeira MJ, Siqueira JTT: Abnormal expression of voltage-gated sodium channels Nav1.7, Nav1.3 and Nav1.8 in trigeminal neuralgia. Neuroscience 164:573-577, 2009

29. Siqueira SRDT, Lara C, Nóbrega JCM, Siqueira JTT, Teixeira MJ: Sensitive evaluation of patients with idiopathic trigeminal neuralgia treated with functional neurosurgery. Pain Clin 18:87-92, 2006

30. Siqueira SRDT, Nóbrega JCM, Teixeira MJ, Siqueira JTT: Olfactory threshold increase in trigeminal neuralgia after balloon compression. Clin Neurol Neurosurg 108:721-725, 2006

31. Siviero M, Teixeira MJ, de Siqueira JTT, Siqueira SRDT: Somesthetic, gustatory, olfactory function and salivary flow in patients with neuropathic trigeminal pain. Oral Dis 16:482-487, 2010
32. Stomal-Słowińska M, Słowiński J, Lee TK, Uitti RJ, Deen $\mathrm{HG}$, Reimer R, et al: Correlation of clinical findings and results of percutaneous balloon compression for patients with trigeminal neuralgia. Clin Neurol Neurosurg 113:14-21, 2011

33. Tomasello F, Alafaci C, Angileri FF, Calisto A, Salpietro FM: Clinical presentation of trigeminal neuralgia and the rationale of microvascular decompression. Neurol Sci 29 Suppl 1:S191-S195, 2008

\section{Author Contributions}

Conception and design: Siqueira, de Almeida, Teixeira, de Siqueira. Acquisition of data: Ichida, de Almeida Nobrega, de Almeida. Analysis and interpretation of data: all authors. Drafting the article: Siqueira. Critically revising the article: Siqueira, Teixeira. Reviewed submitted version of manuscript: Siqueira, de Siqueira. Approved the final version of the manuscript on behalf of all authors: Siqueira. Statistical analysis: Ichida. Administrative/technical/material support: Siqueira, Teixeira, de Siqueira. Study supervision: Siqueira, Teixeira.

\section{Correspondence}

Silvia R. D. T. de Siqueira, Rua Maria Candida, 135 São Paulo, SP 02071-010, Brazil.email: silviadowgan@hotmail.com. 